WellBeing International

WBI Studies Repository

$1-31-2003$

\title{
Play and the Evolution of Fairness: A Game Theory Model
}

Lee Alan Dugatkin

University of Louisville

Marc Bekoff

University of Colorado

Follow this and additional works at: https://www.wellbeingintlstudiesrepository.org/acwp_ena

Part of the Animal Studies Commons, Behavior and Ethology Commons, and the Comparative Psychology Commons

\section{Recommended Citation}

Dugatkin, L. A., \& Bekoff, M. (2003). Play and the evolution of fairness: a game theory model. Behavioural processes, 60(3), 209-214.

This material is brought to you for free and open access by WellBeing International. It has been accepted for inclusion by an authorized administrator of the WBI Studies Repository. For more information, please contact wbisr-info@wellbeingintl.org.

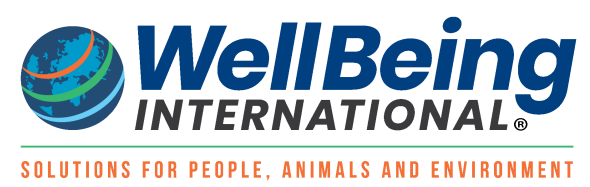




\title{
Play and the Evolution of Fairness: A Game Theory Model
}

\author{
Lee Alan Dugatkin ${ }^{1}$ and Marc Bekoff ${ }^{2}$ \\ ${ }^{1}$ University of Louisville \\ ${ }^{2}$ University of Colorado
}

\section{$\underline{\text { KEYWORDS }}$}

play, fairness, game theory

\begin{abstract}
$\underline{\text { ABSTRACT }}$
Bekoff [J. Consci. Stud. 8 (2001) 81] argued that mammalian social play is a useful behavioral phenotype on which to concentrate in order to learn more about the evolution of fairness. Here, we build a game theoretical model designed to formalize some of the ideas laid out by Bekoff, and to examine whether 'fair' strategies can in fact be evolutionarily stable. The models we present examine fairness at two different developmental stages during an individual's ontogeny, and hence we create four strategies--fair at time 1/fair at time 2, not fair at time 1/not fair at time 2, fair at time 1/not fair at time 2, not fair at time 1/fair at time 2. Our results suggest that when considering species where fairness can be expressed during two different developmental stages, acting fairly should be more common than never acting fairly. In addition, when no one strategy was evolutionarily stable, we found that all four strategies we model can coexist at evolutionary equilibrium. Even in the absence of an overwhelming database from which to test our model, the general predictions we make have significant implications for the evolution of fairness.
\end{abstract}

\section{Introduction}

In a recent paper Bekoff (2001) presented an argument that during social play, while individuals are having fun in a relatively safe environment, they learn ground rules that are acceptable to others (how hard they can bite, how roughly they can interact) and how to resolve conflicts. Bekoff argued that there is a premium on fine-tuning play and playing fairly, and trusting others to do so as well. There are codes of social conduct that regulate what is permissible and what is not permissible during mammalian play, and the existence of these codes might have something to say about the evolution of social morality. For example, individuals might generalize codes of conduct learned when playing with specific individuals early in life to other group members and to other situations. In terms of our own species, Ridley (1996) notes that humans seem to be inordinately upset about unfairness, but we do not know much about other animals' reaction to unfairness.

In social groups individuals may learn what they can and cannot do, and the group's integrity may, to some extent, depend upon individuals having certain rules that regulate their behavior. As a result of 
lessons in social cognition and empathy (Bekoff, 1995, 2000, 2002a,b; Spinka, et al., 2001) that are offered in social play, individuals learn what is acceptable to others.

Play may be a unique category of behavior in which to examine fairness and its ramifications in that asymmetries are tolerated more so than in other social contexts (Biben, 1998; Thompson, 1998; Bekoff, $2001,2002 a, b)$. Play cannot occur if the individuals choose not to engage in the activity, and the equality (or symmetry) needed for play to continue makes it different from other forms of seemingly cooperative behavior (e.g. hunting, care-giving, grooming, food-sharing and defending resources).

From a developmental perspective, behaving fairly during play probably evolved because it helped young animals acquire social (and other) skills needed as they matured into adults. Mammalian social play is a useful behavioral phenotype on which to concentrate in order to learn more about the evolution of fairness and social morality. While birds and individuals of other species engage in social play, there are too few data from which to draw detailed conclusions about the nature of their play.

Numerous mechanisms, such as play invitation signals, variations in the sequencing of actions performed during play when compared with other contexts, self-handicapping, and role-reversing occur during social play in mammals. Here, we build a game theoretical model designed to formalize some of the ideas laid out in Bekoff (2001) and to examine whether 'fair' strategies can in fact be evolutionarily stable.

\section{The model}

Imagine two strategies--behaving fairly (F) and not behaving fairly (NF)--expressed at two points in time, $T_{1}$ and $T_{2} . T_{1}$ might be thought of as that developmental stage during which young individuals engage in play (either fairly or unfairly). $T_{2}$ represents some period of adult life in which F or NF is expressed in a different scenario (e.g. hunting, care giving, grooming, food-sharing and defending resources).

\subsection{Time $T_{1}$}

At time $T_{1}$ we imagine that individuals pair up to play, and each can either choose to play fairly (F) or unfairly (NF). By 'playing fairly' we assume that animals often have social expectations when they engage in various sorts of social encounters, the violation of which constitutes being treated unfairly. For example, when subordinate and dominant individuals engage in play fights, 'self-handicapping' and 'role reversal' often occur (Bekoff and Allen, 1998), during which a dominant individual allows the subordinate individual to act in ways it could easily prevent (e.g. by letting the smaller individual pin it to the ground). Should the dominant switch roles in the middle of such play, and act in a manner inconsistent with role reversal (e.g. by suddenly becoming very aggressive during play), it would be playing in an unfair manner. We assume that the immediate direct fitness consequences of playing fairly versus unfairly are in themselves minor. That is, although play itself may have direct fitness consequences, we assume that the difference between playing fairly and unfairly at $T_{1}$ is minor. However, we assume that individuals who play $\mathrm{F}$ at $T_{1}$ are more adept at playing $\mathrm{F}$ at $T_{2}$. In practice, this assumption translates into individuals learning how to act fairly. While fairness may be expressed in very different contexts at times $T_{1}$ and $T_{2}$, we assume that some general rules associated with acting fairly work across a wide array of contexts such as play, care giving, food sharing, food defense and grooming.

\subsection{Time $T_{2}$}

At $T_{2}, \mathrm{~F}$ or NF is again expressed, and so we now have a total of four strategies that summarize an individual's behavior F/F: (fair at $T_{1}$ and $T_{2}$ ), NF/NF (not fair at times $T_{1}$ and $T_{2}$ ), F/NF (fair at $T_{1}$, unfair at $T_{2}$ ) and NF/F (not fair at $T_{1}$, fair at $T_{2}$ ). We envision that some resource with a total value of $Z$ is available at time $T_{2}$. Fairness at $T_{2}$ refers to the behavior involved in both procuring and dividing a resource. For 
example, imagine that we are studying social carnivores such as pack-living gray wolves, who play as juveniles $\left(T_{1}\right)$ and then communally hunt when adults $\left(T_{2}\right)$. Fairness at $T_{2}$ would imply that a wolf hunts cooperatively and attempts to divide any prey captured equally among hunters. Our discussion of fairness at $T_{1}$ implies that wolves that played fairly as juveniles were better at communally hunting and dividing up prey, as a result of their better familiarity with how to act fairly.

We assume that F/F, F/F pairs obtain resource $z$ with probability 1 , as such individuals played $F$ at $T_{1}$ and are adopting $\mathrm{F}$ at time $T_{2}$. Such individuals learn to play fair at a young age, and then adopt a fair strategy at time $T_{2}$. If neither individual in a pair uses $F$ at time $T_{2}$ (i.e. NF/NF vs. NF/NF; NF/NF vs. F/NF; F/NF vs. F/NF pairings), the probability of the pair obtaining $z$ is set to $h$. All other probabilities of obtaining $z$ are set at:

$$
\begin{aligned}
& \mathrm{F} / \mathrm{NF}, \mathrm{F} / \mathrm{F}=\alpha \\
& \mathrm{NF} / \mathrm{F}, \mathrm{F} / \mathrm{F}=\beta \\
& \mathrm{NT} / \mathrm{NF}, \mathrm{F} / \mathrm{F}=\gamma \\
& \mathrm{F} / \mathrm{NF}, \mathrm{NF} / \mathrm{F}=\delta \\
& \mathrm{NF} / \mathrm{F} . \mathrm{NF} / \mathrm{F}=\varepsilon \\
& \mathrm{NF} / \mathrm{NF}, \mathrm{NF} / \mathrm{F}=\phi
\end{aligned}
$$

where $\alpha>\gamma$ and $\delta>\phi ; \beta>\varepsilon ; \alpha, \beta, \gamma, \delta, \varepsilon, \phi>\eta$.

This rank order weights playing fairly at $T_{1}$ as critically important to obtaining $z$ at time $T_{2}$.

In terms of dividing a resource, we assume that should a pair procure the resource, individuals each get $0.5 z$, if they adopt the same strategy at $T_{2}$. Since fairness dictates splitting the resource, it is clear that two individuals acting fairly at $T_{2}$ split $z$. The reason we assume that two individuals using NF at $T_{2}$ get $0.5 z$ is quite different. NF individuals will attempt to obtain as much of $z$ as possible. However, since we assume that all individuals playing NF at $T_{2}$ are equally likely to succeed at obtaining more than half of $z$, the expected mean for any given individual is $z / 2$. Two individuals playing NF at time $T_{2}$ will be less likely to obtain $z$, but should they procure the resource, in our model, each receives half.

When one individual adopting $\mathrm{F}$ at $T_{2}$ interacts with someone using NF at $T_{2}$, let $j$, the proportion of $z$ an individual using NF (at $T_{2}$ ) receives, where $j>0.5$ (i.e. individuals adopting NF at $T_{2}$ receive a greater portion of $z$ than those who use $F$ ). Our payoff matrix now equals:

\begin{tabular}{lllll}
\hline & F/F & F/NF & NF/F & NF/NF \\
F/F & $0.5 z$ & $(1-j) \alpha z$ & $0.5 \beta z$ & $(1-j) \gamma z$ \\
F/NF & $j \alpha z$ & $0.5 \eta z$ & $j \delta z$ & $0.5 \eta z$ \\
NF/F & $0.5 \beta z$ & $(1-j) \delta z$ & $0.5 \varepsilon z$ & $(1-j) \phi z$ \\
NF/NF & $j \gamma z$ & $0.5 \eta z$ & $j \phi z$ & $0.5 \eta z$ \\
\hline
\end{tabular}

We begin our solution by searching for Evolutionarily Stable Strategies (ESS; Maynard Smith, 1982; Dugatkin and Reeve, 1998), where an ESS is a strategy such that if all individuals adopt it, it can not be invaded by any other strategy (Maynard Smith, 1982). A strategy is an ESS in our model if the diagonal value associated with it (i.e. the payoff of the strategy against itself) is the largest value in its respective 
column. So, for example, in our payoff matrix, neither F/NF nor NF/NF can be an ESS as their diagonal value $(0.5 \eta Z)$ is equal to (but not greater than) another payoff entry in their respective columns.

Analysis of our payoff matrix shows that only F/F can be an ESS, and this is only the case when $\mathrm{j} \alpha<0.5$. When $j \alpha \geq 0.5$, no strategy is an ESS. When no strategy is an ESS, it is possible to use numerical simulations to determine whether some combination of strategies can coexist at a stable equilibrium, and if so, at what frequencies such strategies should equilibrate. To do so let: $p$, frequency of $F / F ; q$, frequency of $\mathrm{F} / \mathrm{NF} ; r$, frequency of NF/F; $s$; frequency of NF/NF $(=1-p-q-s)$.

With these frequencies in hand, we can calculate the payoffs to our four strategies as:

Payoff to F/F

$$
=0.5^{\star} p^{\star} Z+(1-j)^{\star} q^{\star} \alpha^{\star} Z+0.5^{\star} r^{\star} \beta^{\star} Z+(1-j)^{\star} S^{\star} \gamma^{\star} Z
$$

Payoff to F/NF

$$
=p^{\star} j^{\star} \alpha^{\star} z+0.5^{\star} q^{\star} \eta^{\star} z+r^{\star} j^{\star} \delta^{\star} z+0.5^{\star} s^{\star} \eta^{\star} z
$$

Payoff to NF/F

$$
=0.5^{\star} p^{\star} \beta^{\star} Z+(1-J)^{\star} q^{\star} \delta^{\star} Z+0.5^{\star} r^{\star} \varepsilon^{\star} Z+(1-J)^{\star} S^{\star} \phi^{\star} Z
$$

Payoff to NF/NF

$$
=p^{\star} j^{\star} \gamma^{\star} Z+0.5^{\star} q^{\star} \eta^{\star} Z+r^{\star} j^{\star} \phi^{\star} Z+0.5^{\star} S^{\star} \eta^{\star} Z
$$

Once these payoffs are obtained, new frequencies for our strategies for the next iteration (generation) are proportional to payoffs:

$$
\text { Let Total }=\text { Payoff to F/F + Payoff to F/NF + Payoff to NF/F + Payoff to NF/NF }
$$

where $p^{\prime}($ Payoff to F/F)/Total; q'(Payoff to F/NF)/Total; r'(Payoff to NF/F)/Total; s'(Payoff to NF/NF)/Total.

This process is iterated until the frequencies of all four strategies settle down to a stable equilibrium. Exploration of the parameter space where $j \alpha \geq 0.5$, (and hence no strategy is an ESS) demonstrates that all four strategies can coexist at equilibrium (Tables 1 and 2). As such, for this parameter space, all four of our strategies can exist simultaneously, leading to the prediction that there should be considerable variation in fair/unfair behavior in systems where fairness early on and fairness later in life are linked as they are in our model.

\section{Discussion}

Our model demonstrates that F/F can be an ESS, while none of the other three strategies can be. However, perhaps more interesting is the finding that when no strategy is an ESS, all four strategies we modeled can coexist. Since there are no studies we know of in which fairness (or the lack of it) have been measured across numerous points in an individual's ontogeny, more research (both empirical and theoretical) on fairness is needed to test our predictions in detail. Nonetheless, even in the absence of an overwhelming database from which to test our model, the general predictions we make have significant implications for the study of fairness.

In practice, our results translate into two clear predictions. First, always acting fairly should be more common that never acting fairly, when considering species where fairness can be expressed during two 
different developmental stages. Second, we predict that there will be many cases in which none of the strategies we model will be an ESS, but all four will exist at significant frequencies (see Tables 1 and 2). Prior work has shown considerable variability in the tendency to play fairly in early life (Bekoff, 1972, 1977). In addition, work on cooperative hunting - one venue in which fairness might be expressed in late ontogenetic phases - also shows extreme variability in terms of acting fairly or unfairly (Packer and Rutton, 1988; Boesch, 1994). As such, it is not unreasonable to hypothesize that considerable variation should exist in organisms that can choose to be fair or unfair in numerous different contexts throughout their lives. This idea is certainly testable in principle by following individuals and recording how they distribute fairness across many activities as they mature. Lastly, our model predicts that when $\mathrm{j}^{\star} \alpha \geq 0.5$, all four strategies will coexist, otherwise only F/F should be present. Both $j$ and $\alpha$ are measurable in the field ( $j$, for example, by measuring how food is divided when an NF player is involved at $T_{2}$ and $\alpha$, by looking at the success rate of various pairs at $T_{2}$ ), and so it should be possible to predict when $j^{\star} \alpha=0.5$, and hence which of our two solutions (F/F or all strategies) should be in operation in any given system.

Table 1. Results of a numerical analysis when no strategy is an ESS (i.e. when $j \alpha \geq 0.5$ ), $j=0.6, \alpha=0.9, \beta=$ $0.6, \gamma=0.4, \delta=0.4, \varepsilon=0.4, \phi=0.3$ and $\eta=0.2$

\begin{tabular}{|lllll|}
\hline Iteration & Frequency of F/F & Frequency of F/NF & Frequency of NF/F & Frequency of NF/NF \\
\hline$a$ & & & & \\
1 & 0.970 & 0.010 & 0.010 & 0.010 \\
2 & 0.317 & 0.334 & 0.190 & 0.152 \\
3 & 0.365 & 0.268 & 0.207 & 0.161 \\
4 & 0.354 & 0.279 & 0.205 & 0.162 \\
5 & 0.355 & 0.277 & 0.206 & 0.162 \\
6 & 0.355 & 0.277 & 0.206 & 0.162 \\
7 & 0.355 & 0.277 & 0.206 & 0.162 \\
8 & 0.355 & 0.277 & 0.206 & 0.162 \\
9 & 0.355 & 0.277 & 0.206 & 0.162 \\
10 & 0.355 & 0.277 & 0.206 & 0.162 \\
$b$ & & & & \\
1 & 0.010 & 0.970 & 0.010 & 0.010 \\
2 & 0.493 & 0.145 & 0.222 & 0.140 \\
3 & 0.336 & 0.301 & 0.201 & 0.162 \\
4 & 0.359 & 0.273 & 0.206 & 0.161 \\
5 & 0.354 & 0.278 & 0.206 & 0.162 \\
6 & 0.355 & 0.277 & 0.206 & 0.162 \\
7 & 0.355 & 0.277 & 0.206 & 0.162 \\
8 & 0.355 & 0.277 & 0.206 & 0.162 \\
9 & 0.355 & 0.277 & 0.206 & 0.162 \\
10 & 0.355 & 0.277 & 0.206 & 0.162 \\
\hline
\end{tabular}

The value of the resource $(z)$ was set to 1 . Since, however, $z$ appears in all entries in the payoff matrix, changing its value has no effect on the model (i.e. such a change does not preferentially benefit any strategy). F/F was set at an initial frequency of 0.97 , with each of the strategies set at an initial frequency of 0.01 . Here a stable equilibrium containing all four strategies is reached after five generations. (b) Exact same analysis, but with the initial frequency of F/NF set at 0.97 and each of the other strategies set at an initial frequency of 0.01 . This analysis was also run for the case of NF/F at an initial frequency of 0.97 and NF/NF with an initial frequency of 0.97 . In all cases, the end state frequencies are identical to those presented here, demonstrating that these results represent a stable equilibrium. 
Table 2. End state results of numerous numerical analyses when no strategy is an ESS (i.e. when j $\alpha \geq 0.5$ )

\begin{tabular}{|llllll|}
\hline $\boldsymbol{j}$ & $\boldsymbol{\alpha}$ & $\begin{array}{l}\text { Equilibrium } \\
\text { frequency of F/F }\end{array}$ & $\begin{array}{l}\text { Equilibrium } \\
\text { frequency of F/NF }\end{array}$ & $\begin{array}{l}\text { Equilibrium } \\
\text { frequency of NF/F }\end{array}$ & $\begin{array}{l}\text { Equilibrium } \\
\text { frequency of NF/NF }\end{array}$ \\
\hline 0.6 & 0.9 & 0.355 & 0.277 & 0.205 & 0.161 \\
0.7 & 0.8 & 0.325 & 0.294 & 0.195 & 0.186 \\
0.7 & 0.9 & 0.324 & 0.307 & 0.189 & 0.180 \\
0.8 & 0.7 & 0.294 & 0.309 & 0.182 & 0.214 \\
0.8 & 0.8 & 0.293 & 0.324 & 0.176 & 0.201 \\
0.8 & 0.9 & 0.291 & 0.339 & 0.170 & 0.199 \\
0.9 & 0.6 & 0.264 & 0.324 & 0.166 & 0.246 \\
0.9 & 0.7 & 0.258 & 0.342 & 0.161 & 0.239 \\
0.9 & 0.8 & 0.255 & 0.359 & 0.155 & 0.232 \\
0.9 & 0.9 & 0.251 & 0.374 & 0.150 & 0.225 \\
\hline
\end{tabular}

$z=1, \beta=0.6, \gamma=0.4, \delta=0.4, \varepsilon=0.4, \phi=0.3$ and $\eta=0.2$. Analyses were also run for the case of $F / F$ having an initial frequency of 0.97 , F/NF at an initial frequency of 0.97 , NF/F at initial frequency of 0.97 and NF/NF with an initial frequency of 0.97 . In all cases, the end state frequencies are identical to those presented here, demonstrating that these results represent a stable equilibrium.

Future comparative research that considers the nature and details of the social exchanges that are needed for animals to engage in play will undoubtedly produce data that bear on the questions that we raise here and also help to 'operationalize' the notion of behaving fairly by informing us about what sorts of evidence confirm that animals are behaving with some sense of fairness.

Learning about the taxonomic distribution of animal morality involves answering numerous and often difficult questions. Perhaps it will turn out that the best explanation for existing data in various taxa is that some individuals do indeed (on some occasions) modify their behavior to cooperate and to interact fairly (Okamoto and Matsumura, 2001). This sort of egalitarianism may be a precondition for the evolution of social morality in humans. We sincerely hope that models such as ours will stimulate more empirical research into the evolution of fairness.

\section{References}

Bekoff, M., 1972. The development of social interaction, play, and metacommunication in mammals: an ethological perspective. Q. Rev. Biol. 47, 412-434.

Bekoff, M., 1977. Mammalian dispersal and the ontogeny of individual behavioral phenotypes. Am. Nat. 111, 715-732.

Bekoff, M., 1995. Play signals as punctuation: the structure of social play in canids. Behaviour 132, 419429.

Bekoff, M., 2000. Animal emotions: exploring passionate natures. BioScience 50, 861-870.

Bekoff, M., 2001. Social play behavior: cooperation, fairness, trust, and the evolution of morality. J. Consci. Stud. 8, 81-90.

Bekoff, M., 2002. Virtuous nature. New Scientist 13, 34-37.

Bekoff, M., 2002. Minding Animals: Awareness, Emotions and Heart. Oxford University Press, New York.

Bekoff, M., Allen, C., 1998. Intentional communication and social play: how and why animals negotiate and agree to play. In: Bekoff, M., Byers, J.A. (Eds.), Animal Play: Evolutionary, Comparative, and Ecological Perspectives. Cambridge University Press, Cambridge, pp. 97-114. 
Biben, M., 1998. Squirrel monkey playfighting: making the case for a cognitive training function for play. In: Bekoff, M., Byers, J.A. (Eds.), Animal Play. Evolutionary, Comparative and Ecological Perspectives. Cambridge University Press, Cambridge, pp. 161-182.

Boesch, C., 1994. Cooperative hunting in wild chimpanzees. Anim. Behav. 48, 653-667.

Dugatkin, L.A., Reeve, H.K. (Eds.), Game Theory and Animal Behavior. Oxford University Press, New York 1998.

Maynard Smith, J., 1982. Evolution and the Theory of Games. Cambridge University Press, Cambridge.

Okamoto, K., Matsumura, S., 2001. The evolution of punishment and apology: an iterated prisoner's dilemma model. Evol. Ecol. 14, 703-720.

Packer, C., Rutton, L., 1988. The evolution of cooperative hunting. Am. Nat. 132, 159-194.

Ridley, M., 1996. The Origins of Virtue: Human Instincts and the Evolution of Cooperation. Viking, New York.

Spinka, M., Newberry, R.C., Bekoff, M., 2001. Mammalian play: training for the unexpected. Q. Rev. Biol. 76, 141-168.

Thompson, K.V., 1998. Self assessment in juvenile play. In: Bekoff, M., Byers, J.A. (Eds.), Animal Play. Evolutionary, Comparative and Ecological Perspectives. Cambridge University Press, Cambridge, pp. 183-204. 